\title{
Inhibitory Effect and Possible Mechanism of Intraurethral Stimulation on Overactive Bladder in Female Rats
}

\author{
Yu Tian ${ }^{1,2,3}$, Limin Liao ${ }^{3}$, Jean Jacques Wyndaele ${ }^{1,2}$ \\ ${ }^{1}$ Department Translational Neurosciences, Laboratory Urology, University of Antwerp, Faculty GGW, Antwerp, Belgium \\ ${ }^{2}$ Department of Urology, University Hospital Antwerp, Antwerp, Belgium \\ ${ }^{3}$ Department of Urology, China Rehabilitation Research Center, Capital Medical University, Beijing, China
}

Purpose: To investigate the inhibitory effect and possible mechanism of intraurethral stimulation on overactive bladder $(\mathrm{OAB})$ induced by acetic acid irritation.

Methods: Cystometry was performed in 13 urethane-anesthetized female rats. Intravesical infusion of $0.5 \%$ acetic acid was used to irritate the bladder and induce OAB. Multiple cystometrograms were performed with mirabegron, continuous stimulation, mirabegron plus continuous stimulation, and $\beta 3$-adrenoceptor antagonist plus continuous stimulation to determine the mechanism underlying the inhibitory effect by intraurethral stimulation.

Results: Infusion of acetic acid significantly decreased bladder capacity. Intraurethral stimulation at $2.5 \mathrm{~Hz}$ plus mirabegron significantly increased bladder capacity and decreased the nonvoiding contraction count. The changes were strongly inhibited after the $\beta 3$-adrenoceptor antagonist was administered.

Conclusions: Activation of urethral afferent nerves can reverse OAB, which activates $\mathrm{C}$-fiber afferent nerves. This animal study indicates that intraurethral stimulation may interfere with $\mathrm{OAB}$ through hypogastric nerve activation and pudendal nerve neuromodulation.

Keywords: Urinary Bladder, Overactive; Splanchnic Nerves; Electric Stimulation; Rats

- Fund Support: The study was done with financial support of the Trust Marie Curie project and the research fund of the Urologic Department, University Hospital Antwerp.

- Research Ethics: Animal care and experimental procedures were reviewed and approved by the Institutional Animal Care and Use Committee of Antwerp University (code: 2013-50).

- Conflict of Interest: No potential conflict of interest relevant to this article was reported.

\section{INTRODUCTION}

Overactive bladder $(\mathrm{OAB})$ symptoms include frequency, urgency, and incontinence and are difficult to manage with medi- cation alone [1,2]. The symptoms have a substantial impact on all aspects of patient well-being and reduce quality of life. Sacral neuromodulation has been used to treat patients who are refractory to other therapies $[3,4]$. Currently, a tined lead elec-

Corresponding author: Limin Liao (iD http://orcid.org/0000-0002-7092-6576

Department of Urology, China Rehabilitation Research Center, Capital Medical

University, No. 10, Jiaomen Beilu, Beijing 100068, China

E-mail: Imliao@263.net / Tel: +86-13-311268064 / Fax: +86-10-67570492

Co-Corresponding Author: Jean Jacques Wyndaele (i) http://orcid.org/0000-0002-0879-6854

Department of Urology, Antwerp University, 10 Wilrijkstraat, 2650 Edegem, Antwerp, Belgium

E-mail: wyndaelejj@skynet.be / Tel: +32-3821-3511 / Fax: +32-3821-4479

Submitted: July 12, 2015 / Accepted: August 27, 2015 This is an Open Access article distributed under the terms of the Cre-
ative Commons Attribution Non-Commercial License (http://creative-
commons.org/licenses/by-nc/3.0/) which permits unrestricted non-commercial use, distri-
bution, and reproduction in any medium, provided the original work is properly cited. 
trode needs to be permanently implanted in the S3 sacral foramen in addition to an implanted stimulator (InterStim stimulator, Medtronic Inc., Parkway Minneapolis, MN, USA) [5,6]. Importantly, sacral neuromodulation has been associated with different levels of effectiveness [7].

A recent study indicated that pudendal neuromodulation may be as effective as sacral neuromodulation in treating $\mathrm{OAB}$ [8]. Moreover, pudendal neuromodulation has attracted significant attention because it can be applied to specific areas in the pelvic region, including the vagina, anus, and urethra [9]. Activation of afferents in the sensory pudendal nerve can reflexively induce efferent firing in the pudendal nerve to elicit sphincter muscle contractions that in turn induce pudendal afferent firing via motor-sensory coupling $[10,11]$. That electrically evoked reflex that inhibits $\mathrm{OAB}$ has never been examined in the rat $[12,13]$.

In this study, we describe the inhibitory effects of intraurethral stimulation or neuromodulation on acetic acid (AA)-induced bladder irritation or $\mathrm{OAB}$ in the rat. The outcomes of stimulation with or without drugs were compared, and the possible mechanism underlying the inhibitory effect was determined.

\section{MATERIALS AND METHODS}

The experiments were performed on 13 virgin female Sprague
Dawley rats (220-250 g). The animals were anesthetized by intraperitoneal injection of urethane $(1.5 \mathrm{~g} / \mathrm{kg})$. The rats in this study were not awakened during the procedures. Animal care and experimental procedures were reviewed and approved by the Institutional Animal Care and Use Committee of Antwerp University (code 2013-50).

\section{Experiment Setup}

A ventral midline abdominal incision was made, and the bladder was catheterized via the bladder dome with a PE-50 polyethylene catheter (Clay Adams, Parsippany, NJ, USA). The catheter was connected via a three-way valve to a pressure transducer (emka TECHNOLOGIES, Paris, France), an NE1000 syringe pump (New Era Pump Systems, Farmingdale, NY, USA), and an exit port to empty the bladder.

A 20-gauge intravenous catheter (Becton-Dickinson, Madrid, Spain) was inserted into the urethra via the urethral meatus. Two coated platinum wires, which were 9 and $7 \mathrm{~mm}$ longer than the catheter (Fig. 1A), were fully inserted into the catheter, and then the catheter together with the wires was moved outward until the catheter was nearly out of the urethra. The wires were now positioned in the urethra 9 and $7 \mathrm{~mm}$ from the meatus, thus permitting bipolar stimulation in the urethral lumen (Fig. 1B). The urethral meatus was tied around the wires with 3-0 silk to prevent leakage and hold the electrodes in place (Fig. 1C).
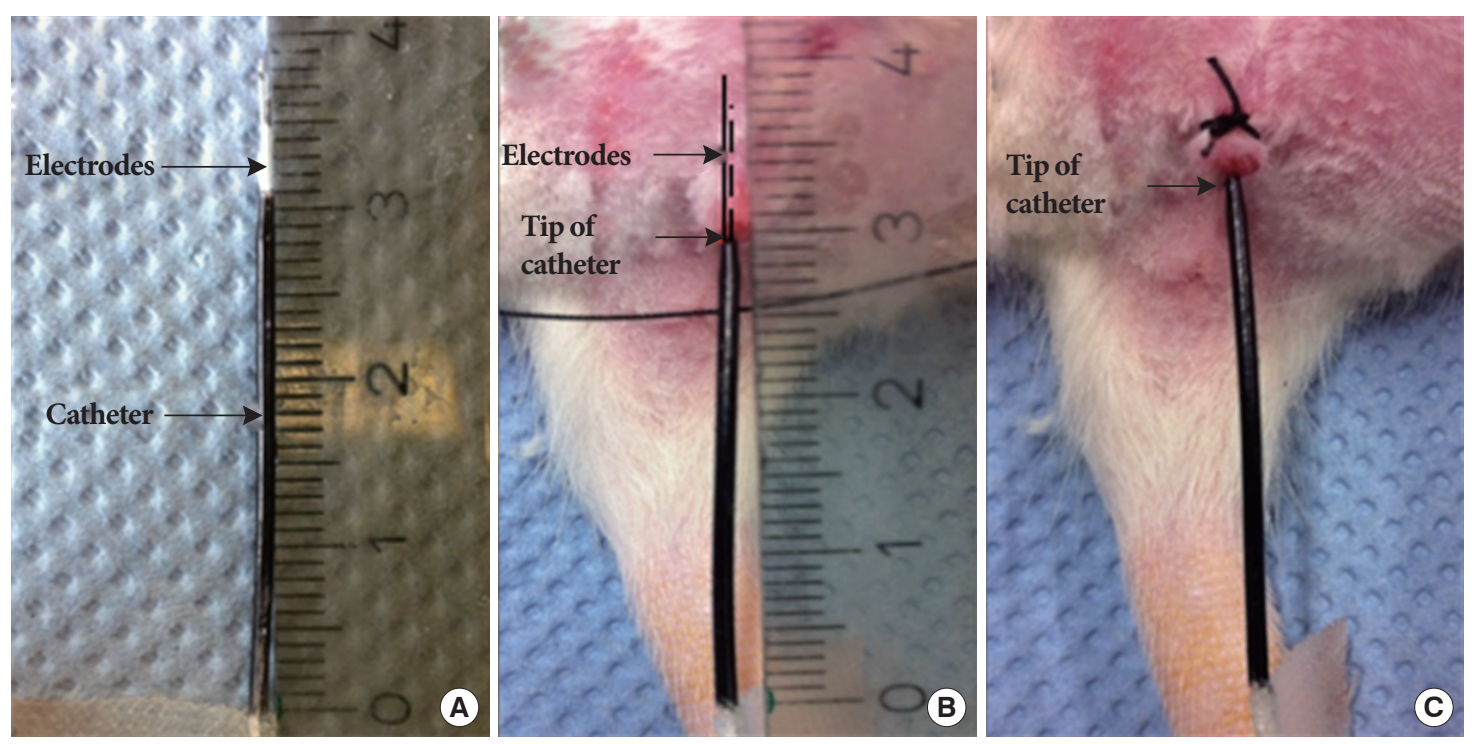

Fig. 1. Electrode setup and location. (A) Two coated platinum wires were 9 and $7 \mathrm{~mm}$ longer than the catheter. (B) The electrodes were fully inserted into the catheter. The catheter and the wires were moved outward together until the catheter was nearly out of the urethra. (C) The urethral meatus was tied around the wires with 3-0 silk to prevent leakage and hold the two electrodes in place. 
A test electrical stimulation in preliminary studies used uniphasic rectangular square wave pulses at $0.5,2,3,5,10,20,100$, 200 , and $1,000 \mathrm{~Hz}$. The results indicated that the stimulation frequency between 2 and $3 \mathrm{~Hz}$ inhibited bladder contractions and increased bladder capacity $(B C)$ in normal rats $(n=9)$. The minimum current amplitude to elicit a urethral external sphincter response was known to range from 0.01 to $0.05 \mathrm{~mA}$ [14]. In our study, a current amplitude of $0.06 \mathrm{~mA}$ was applied to activate the urethral afferent nerves during intraurethral stimulation at 2.5 $\mathrm{Hz}$. Inhibitory actions on bladder activity under these conditions were evaluated.

The bladder was filled with saline or $0.5 \% \mathrm{AA}$ in all experiments $(n=13)$ at room temperature at a rate of $0.09 \mathrm{~mL} / \mathrm{min}$. The $\mathrm{BC}$ was defined as the bladder volume at which voiding contraction ( $>25 \mathrm{~cm} \mathrm{H}_{2} \mathrm{O}$ ) was induced. Nonvoiding contraction (NVC) was defined as a bladder contraction $>5 \mathrm{~cm} \mathrm{H}_{2} \mathrm{O}$ from the baseline pressure before voiding contraction. The bladder was emptied after each cystometrogram, and a 20- to 25 -minute rest period was permitted to allow the bladder reflexes to recover.

\section{Protocols}

To begin, 2 or 3 control cystometries with saline filling were performed. When at least 2 stable control measurements were obtained, another 3-5 intermittent filling cystometries were performed within the following 30-60 minutes with a $0.5 \%$ AA infusion to induce bladder irritation and overactivity. The urethra was electrically stimulated, and the effect of the stimulation on the OAB was studied. To determine the inhibitory mechanism of intraurethral stimulation, we evaluated the effects of stimulation in the presence of a $\beta 3$-adrenoceptor agonist (mirabegron, $0.1 \mathrm{mg} / \mathrm{kg}$ ) and a $\beta 3$-adrenoceptor antagonist (L748337, $0.1 \mathrm{mg} / \mathrm{kg}$ ). Mirabegron, continuous stimulation (CS) alone, mirabegron plus CS, and L748337 plus CS were applied separately during 3 sequential cystometrograms. Bladder filling was performed 3 minutes after administration of mirabegron or L748337. At the end of the experiment, another cystometrogram with AA infusion was performed without stimulation or drugs to determine whether the irritation-induced micturition reflex returned to the prestimulation condition.

\section{Solutions and Drugs}

N, N-dimethylacetamide (DMA), urethane, AA, cremophor, L748337 (100 nM), and mirabegron ( $1 \mathrm{mg} / \mathrm{kg}$ ) were purchased from Sigma-Aldrich (St. Louis, MO, USA). Mirabegron was dissolved in 5\% DMA, 5\% cremophor, and 90\% distilled water. Drugs and vehicle were administrated intravenously through a polyethylene catheter (PE-50) that was placed in the right external jugular vein.

\section{Data Analysis}

Statistical analysis was performed using IBM SPSS Statistics ver. 19.0 (IBM Co., Armonk, NY, USA). The maximum pressure of the voiding contraction (MPC), BC, and NVC count were measured. Repeated measurements in the same animal during the same experiment were averaged to avoid large variation caused by individual animal differences. All reported values were the mean \pm standard error. One-way analysis of variance was used to detect statistical significance. For all statistical tests, a $\mathrm{P}<0.05$ was considered significant.

\section{RESULTS}

The BC decreased to $28.0 \%(0.10 \pm 0.04 \mathrm{~mL})$ of the saline control capacity $(0.36 \pm 0.05 \mathrm{~mL})$ after infusing $0.5 \%$ AA. Mirabegron, CS, and mirabegron plus CS significantly increased the $\mathrm{BC}$ to $174.2 \% \pm 27.9 \%(0.17 \pm 0.05 \mathrm{~mL}), 175.4 \% \pm 30.2 \%$ $(0.17 \pm 0.04 \mathrm{~mL})$, and $246.2 \% \pm 46.7 \%(0.23 \pm 0.04 \mathrm{~mL})$, respectively, of the AA control capacity (Figs. 2, 3A). The BC in the mirabegron plus CS group was significantly increased compared with that in the CS-alone group $(\mathrm{P}=0.006)$ (Fig. 3B). There was no significant difference in the $\mathrm{BC}$ between the mirabegron and CS groups $(\mathrm{P}=0.950)$ (Fig. 3B). Also, there was no significant difference between the L748337 plus CS group

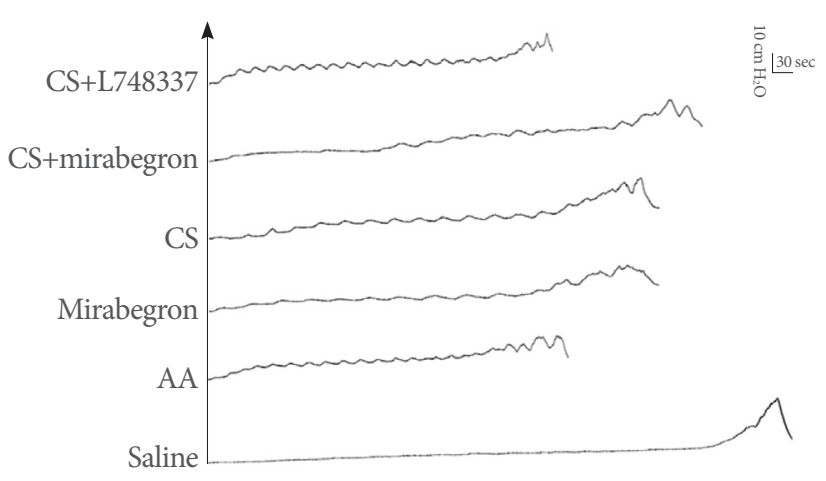

Fig. 2. Examples of cystometries with infusion of saline, acetic acid (AA), mirabegron, continuous stimulation (CS), mirabegron plus CS, L748337 plus CS, and AA. Bladder capacity decreased after infusing 0.5\% AA. Mirabegron, CS, and mirabegron plus CS increased bladder capacity. 

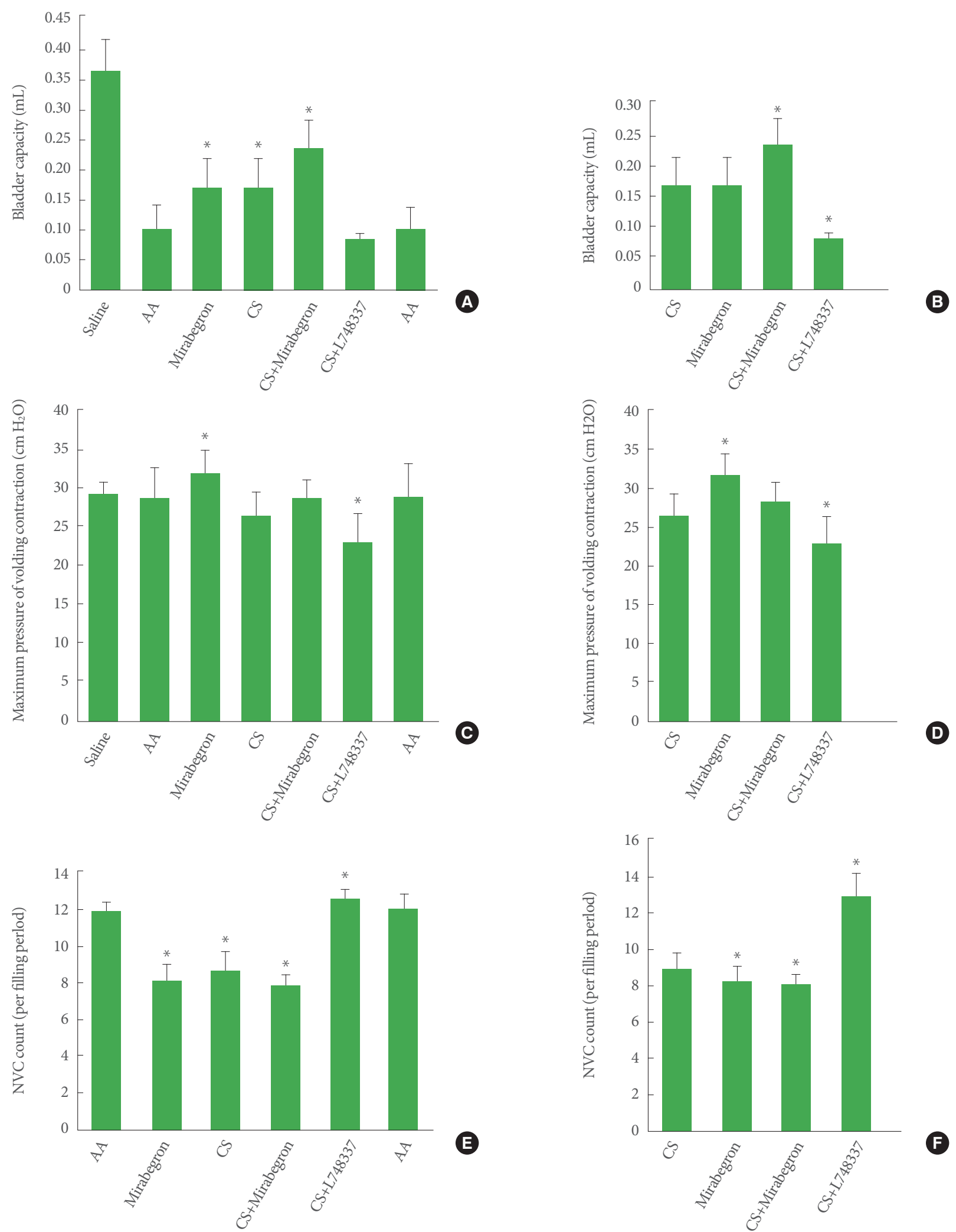

Fig. 3. Results with infusion of saline, acetic acid (AA), mirabegron, continuous stimulation (CS), mirabegron plus CS, L748337 plus $\mathrm{CS}$, and AA. (Continued to the next page) 
Fig. 3. (Continued) (A) mirabegron, CS, and mirabegron plus CS significantly increased bladder capacity (BC) after AA. (B) The BC of the mirabegron $(\mathrm{P}=0.005)$ and $\mathrm{CS}$ groups $(\mathrm{P}=0.003)$ was significantly increased. There was no significant difference in $\mathrm{BC}$ between the mirabegron and CS groups $(\mathrm{P}=0.964)$. (C) The maximum pressure of voiding contraction (MPC) increased after mirabegron $(\mathrm{P}=0.014)$. The MPC decreased in the $\mathrm{L} 748337$ plus CS group $(\mathrm{P}=0.004)$. (D) The MPC of the CS $(\mathrm{P}=0.947)$ and mirabegron plus CS $(\mathrm{P}=0.980)$ groups was not significantly different compared with AA. (E) The nonvoiding contraction (NVC) count per filling period in the mirabegron $(\mathrm{P}=0.003)$, CS $(\mathrm{P}=0.001)$, and mirabegron plus CS groups $(\mathrm{P}=0.008)$ was decreased. The NVC count was increased after $\mathrm{L} 748337(\mathrm{P}=0.017)$. (F) The ability of mirabegron to inhibit NVC was more significant than that of CS $(\mathrm{P}=0.028)$. ${ }^{\star} \mathrm{P}<0.05$, statistically significant difference.

and the AA control group $(\mathrm{P}=0.294)$ (Fig. 3A).

The MPC in OAB rats increased from $28.38 \pm 3.83 \mathrm{~cm} \mathrm{H}_{2} \mathrm{O}$ to $31.5 \pm 2.92 \mathrm{~cm} \mathrm{H}_{2} \mathrm{O}$ after mirabegron administration $(\mathrm{P}=0.014)$ (Fig. 3C). The MPC decreased to $22.78 \pm 3.65 \mathrm{H}_{2} \mathrm{O}$ in the group with $\mathrm{L} 748337$ plus CS $(\mathrm{P}=0.004)$ (Fig. 3C). The MPCs of the CS $(\mathrm{P}=0.947)$ and mirabegron plus CS groups $(\mathrm{P}=0.980)$ were not significantly different compared with those in the AA control group (Fig. 3D).

The NVC counts per filling period in the mirabegron, CS, and mirabegron plus CS groups were decreased from 11.97 \pm 0.40 to $8.1 \pm 0.89(\mathrm{P}=0.003), 8.69 \pm 1.03(\mathrm{P}=0.001)$, and $7.95 \pm 0.51(\mathrm{P}=0.008)$, respectively (Fig. $3 \mathrm{E})$. The NVC count was increased from $8.69 \pm 1.0$ to $12.62 \pm 0.5$ after L748337 administration $(\mathrm{P}=0.017)$ (Fig. 3E). The ability of mirabegron to inhibit NVC was more significant than that of CS $(\mathrm{P}=0.028)$ (Fig. 3F).

\section{DISCUSSION}

This study showed that intraurethral stimulation at $2.5 \mathrm{~Hz}$ effectively suppressed bladder overactivity induced by AA infusion in rats.

Bladder activity is induced primarily by activation of bladder A $\delta$-fiber afferents during saline filling; however, after AA irritation, bladder $\mathrm{C}$-fiber afferents were sensitized and induced $\mathrm{OAB}$, including a marked decrease in BC (Fig. 2) [6]. Bladder irritation induced a micturition reflex, which depends on a normal reflex pathway activated by $\mathrm{A} \delta$-fibers but was facilitated by $\mathrm{C}$-fiber afferent input. Thus, it is difficult to determine whether intraurethral stimulation specifically reversed C-fiber afferent-induced facilitation or suppressed the A $\delta$-fiber activated pathway.

Intraurethral stimulation at $2.5 \mathrm{~Hz}$ may activate the hypogastric and pudendal nerves to modulate bladder activities. After blocking of the $\beta 3$-adrenergic bladder pathway with L748337, the ability to increase the BC was strongly decreased (Figs. 2, 3A). L748337 also effectively reversed intraurethral stimulation- induced changes in the NVC count (Fig. 2). Those results suggest that intraurethral stimulation depends on activation of hypogastric efferent nerves to inhibit bladder activity. In addition, the midurethra, where the external urethral striated muscle sphincter is located, also contains smooth muscle layers innervated by the hypogastric nerve $[15,16]$.

Previous studies have demonstrated that low-frequency pudendal trunk stimulation evokes a robust reflex activation of hypogastric efferents [12,17]; however, the ability to increase $\mathrm{BC}$ was preserved or recovered when the stimulation intensity was increased following hypogastric transection [12]. These results suggest that intraurethral stimulation at $2.5 \mathrm{~Hz}$ and lowfrequency pudendal trunk stimulation may have different mechanisms by which bladder activities are modulated.

Both CS and mirabegron have been shown to increase BC. Furthermore, there was no significant difference in $\mathrm{BC}$ between mirabegron and CS (Fig. 3B); however, MPC was increased after mirabegron administration. MPCs in the CS group were not significantly different from those in the AA control group (Fig. 3C). That result indicates that CS has the ability to decrease MPC. A previous study [18], which showed that pudendal sensory branch transection reduced maximum bladder pressure, suggested that intraurethral stimulation decreased MPC by activating the pudendal nerve. The external urethral sphincter, which is innervated by the pudendal nerve, could be directly modulated by the stimulation. The urethral length measured from the bladder to the urethral meatus is $13.0-15.8 \mathrm{~mm}$ in virgin female Sprague Dawley rats (170-200 g). The peak in the striated muscle area is located $7.8-10.8 \mathrm{~mm}$ from the ureteral orifice [19]. The distance from the anode to the urethral meatus was about $9 \mathrm{~mm}$ in our experiments, and thus the electrodes were positioned in the striated muscle area. Intraurethral electrical stimulation at the midurethral portion stimulated the hypogastric and pudendal nerves, which also innervate the bladder.

Therefore, activation of the hypogastric (afferent)-to-hypogastric (efferent) and pudendal (afferent)-to-hypogastric (effer- 
ent) reflex pathways could be the mechanisms of action underlying intraurethral electrical stimulation.

Furthermore, recent studies in both chronic spinal cord-injured and spinal-intact animals showed that pudendal nerve stimulation inhibits bladder activity or excites the bladder, depending on stimulation frequency $[20,21]$. Those results arouse our interest in the longtime effect of intraurethral stimulation in spinal cord-injured animals.

Clinical trials have shown that the optimal stimulus parameters for neuromodulation therapy in human subjects are probably different from those obtained in this animal study; however, the testing protocol and the results of this preclinical study have provided useful information for the design of a clinical trial in humans with preserved hypogastric innervation.

In conclusion, we studied the inhibitory effect of $2.5-\mathrm{Hz}$ intraurethral stimulation in $\mathrm{OAB}$ rats. Although many questions about intraurethral stimulation remain to be answered, this animal study indicated that intraurethral stimulation may inhibit $\mathrm{OAB}$ through hypogastric nerve activation and pudendal nerve neuromodulation.

\section{ACKNOWLEDGEMENTS}

The authors thank Stefan De Wachter, Michel Wyndaele, and Sara Persyn for assistance with the experimental setup and preparation. The authors also thank the National Natural Scientific Foundation of China (81270847) for assistance during preparation for the publication of this manuscript.

\section{REFERENCES}

1. Lofgren OE. Re: Overactive bladder: a better understanding of pathophysiology, diagnosis and management. A. J. Wein and R. R. Rackley J Urol, suppl., 2006; 175: S5-S10. J Urol 2007;178:1553; author reply 1553.

2. Andersson KE, Wein AJ. Pharmacology of the lower urinary tract: basis for current and future treatments of urinary incontinence. Pharmacol Rev 2004;56:581-631.

3. Bosch JL, Groen J. Sacral nerve neuromodulation in the treatment of patients with refractory motor urge incontinence: long-term results of a prospective longitudinal study. J Urol 2000;163:1219-22.

4. van Kerrebroeck PE, van Voskuilen AC, Heesakkers JP, Lycklama a Nijholt AA, Siegel S, Jonas U, et al. Results of sacral neuromodulation therapy for urinary voiding dysfunction: outcomes of a prospective, worldwide clinical study. J Urol 2007;178:2029-34.
5. Kessler TM, Buchser E, Meyer S, Engeler DS, Al-Khodairy AW, Bersch U, et al. Sacral neuromodulation for refractory lower urinary tract dysfunction: results of a nationwide registry in Switzerland. Eur Urol 2007;51:1357-63.

6. Fowler CJ, Griffiths D, de Groat WC. The neural control of micturition. Nat Rev Neurosci 2008;9:453-66.

7. Brazzelli M, Murray A, Fraser C. Efficacy and safety of sacral nerve stimulation for urinary urge incontinence: a systematic review. J Urol 2006;175(3 Pt 1):835-41.

8. Peters KM, Feber KM, Bennett RC. Sacral versus pudendal nerve stimulation for voiding dysfunction: a prospective, single-blinded, randomized, crossover trial. Neurourol Urodyn 2005;24:643-7.

9. Martin WD, Fletcher TF, Bradley WE. Innervation of feline perineal musculature. Anat Rec 1974;180:15-29.

10. Lagunes-Cordoba R, Hernandez PR, Raya JG, Munoz-Martinez EJ. Functional coupling between motor and sensory nerves through contraction of sphincters in the pudendal area of the female cat. J Neurophysiol 2010;103:74-82.

11. Fowler CJ, Swinn MJ, Goodwin RJ, Oliver S, Craggs M. Studies of the latency of pelvic floor contraction during peripheral nerve evaluation show that the muscle response is reflexly mediated. J Urol 2000;163:881-3.

12. Woock JP, Yoo PB, Grill WM. Mechanisms of reflex bladder activation by pudendal afferents. Am J Physiol Regul Integr Comp Physiol 2011;300:R398-407.

13. Woock JP, Yoo PB, Grill WM. Intraurethral stimulation evokes bladder responses via 2 distinct reflex pathways. J Urol 2009;182:366-73.

14. Chen SC, Grill WM, Fan WJ, Kou YR, Lin YS, Lai CH, et al. Bilateral pudendal afferent stimulation improves bladder emptying in rats with urinary retention. BJU Int 2012;109:1051-8.

15. Elbadawi A, Atta MA. Ultrastructure of vesicourethral innervation: IV. Evidence for somatomotor plus autonomic innervation of the male feline rhabdosphincter. Neurourol Urodyn1985;4:23-36.

16. Lim SH, Wang TJ, Tseng GF, Lee YF, Huang YS, Chen JR, et al. The distribution of muscles fibers and their types in the female rat urethra: cytoarchitecture and three-dimensional reconstruction. Anat Rec (Hoboken) 2013;296:1640-9.

17. Craggs M, McFarlane J. Neuromodulation of the lower urinary tract. Exp Physiol 1999;84:149-60.

18. Peng CW, Chen JJ, Cheng CL, Grill WM. Role of pudendal afferents in voiding efficiency in the rat. Am J Physiol Regul Integr Comp Physiol 2008;294:R660-72.

19. Kim RJ, Kerns JM, Liu S, Nagel T, Zaszczurynski P, Lin DL, et al. Striated muscle and nerve fascicle distribution in the female rat urethral sphincter. Anat Rec (Hoboken) 2007;290:145-54. 
20. Tai C, Wang J, Wang X, de Groat WC, Roppolo JR. Bladder inhibition or voiding induced by pudendal nerve stimulation in chronic spinal cord injured cats. Neurourol Urodyn 2007;26:570-7.
21. Boggs JW, Wenzel BJ, Gustafson KJ, Grill WM. Frequency-dependent selection of reflexes by pudendal afferents in the cat. J Physiol 2006;577(Pt 1):115-26. 